\title{
Reappearance of Salmonella serovar Choleraesuis var. Kunzendorf in Danish pig herds
}

Pedersen, Karl; Sørensen, Gitte; Löfström, Charlotta; Leekitcharoenphon, Pimlapas; Nielsen, bent; Wingstrand, Anne; Aarestrup, Frank Møller; Hendriksen, Rene S.; Baggesen, Dorte Lau

Published in:

Veterinary Microbiology

Link to article, DOI:

10.1016/j.vetmic.2015.01.004

Publication date:

2015

Link back to DTU Orbit

Citation $(A P A)$ :

Pedersen, K., Sørensen, G., Löfström, C., Leekitcharoenphon, P., Nielsen, B., Wingstrand, A., Aarestrup, F. M., Hendriksen, R. S., \& Baggesen, D. L. (2015). Reappearance of Salmonella serovar Choleraesuis var.

Kunzendorf in Danish pig herds. Veterinary Microbiology, 176(3-4), 282-291.

https://doi.org/10.1016/j.vetmic.2015.01.004

\section{General rights}

Copyright and moral rights for the publications made accessible in the public portal are retained by the authors and/or other copyright owners and it is a condition of accessing publications that users recognise and abide by the legal requirements associated with these rights.

- Users may download and print one copy of any publication from the public portal for the purpose of private study or research.

- You may not further distribute the material or use it for any profit-making activity or commercial gain

- You may freely distribute the URL identifying the publication in the public portal 


\section{Accepted Manuscript}

Title: Reappearance of Salmonella serovar Choleraesuis var. Kunzendorf in Danish pig herds

Author: Karl Pedersen Gitte Sørensen Charlotta Löfström Pimlapas Leekitcharoenphon Bent Nielsen Anne Wingstrand Frank M. Aarestrup René S. Hendriksen Dorte Lau Baggesen

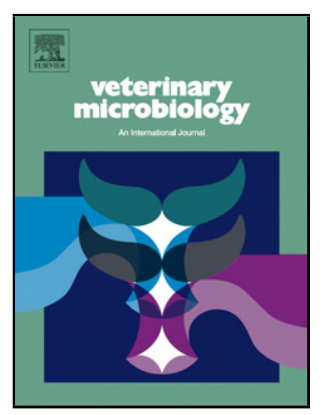

PII:

DOI:

Reference: S0378-1135(15)00006-1

To appear in: $\quad$ VETMIC

Received date: $\quad$ 14-5-2014

Revised date: $\quad 30-12-2014$

Accepted date: $\quad$ 5-1-2015

Please cite this article as: Pedersen, K., Sorensen, G., Löfström, C., Leekitcharoenphon, P., Nielsen, B., Wingstrand, A., Aarestrup, F.M., Hendriksen, R.S., Baggesen, D.L.,Reappearance of Salmonella serovar Choleraesuis var. Kunzendorf in Danish pig herds, Veterinary Microbiology (2015), http://dx.doi.org/10.1016/j.vetmic.2015.01.004

This is a PDF file of an unedited manuscript that has been accepted for publication. As a service to our customers we are providing this early version of the manuscript. The manuscript will undergo copyediting, typesetting, and review of the resulting proof before it is published in its final form. Please note that during the production process errors may be discovered which could affect the content, and all legal disclaimers that apply to the journal pertain. 
Reappearance of Salmonella serovar Choleraesuis var. Kunzendorf in Danish pig herds

3 Karl Pedersen ${ }^{\mathrm{a},{ }^{*}}$, Gitte Sørensen ${ }^{\mathrm{b}}$, Charlotta Löfström ${ }^{\mathrm{b}}$, Pimlapas Leekitcharoenphon ${ }^{\mathrm{c}}$, Bent

4 Nielsen $^{\mathrm{d}}$, Anne Wingstrand ${ }^{\mathrm{b}}$, Frank M. Aarestrup ${ }^{\mathrm{c}}$, René S. Hendriksen ${ }^{\mathrm{c}}$, Dorte Lau Baggesen ${ }^{\mathrm{b}}$

$5 \quad{ }^{a}$ Technical University of Denmark, National Veterinary Institute, Frederiksberg C, Denmark, ${ }^{b}$

6 Technical University of Denmark, National Food Institute, Søborg, Denmark, ${ }^{c}$ Technical

7 University of Denmark, National Food Institute, Kongens Lyngby, Denmark, ${ }^{d}$ Danish Agriculture

8 and Food Council, Pig Research Centre, Axelborg, Copenhagen V, Denmark

9

* Corresponding author

11 Karl Pedersen

12 Technical University of Denmark

13 National Veterinary Institute

14 Bülowsvej 27

15 DK-1870 Frederiksberg C

16 Denmark

17 Phone +45 35886201

18

E-mail kape@vet.dtu.dk

19 


\section{Abstract}

Salmonella enterica serovar Choleraesuis is a porcine adapted serovar which may cause serious outbreaks in pigs. Here we describe outbreaks of salmonellosis due to $S$. Choleraesuis in four Danish pig farms in 2012 - 2013 by clinic, serology, and microbiology and compare the isolates to those of a previous outbreak in 1999 - 2000. The infection was in some herds associated with high mortality and a moderate to high sero-prevalence was found. In 2012 - 2013 the disease contributed to increased mortality but occurred concomitant with other disease problems in the herds, which likely delayed the diagnosis by up to several months. Nine isolates from the four farms in 2012 2013 and 14 isolates obtained from the outbreak in Denmark in 1999 - 2000 were subjected to typing using pulsed-field gel electrophoresis (PFGE). Seven isolates were selected for whole genome sequencing (WGS). The PFGE results of 23 isolates displayed five different profiles. The isolates from 2012 - 2013 revealed two distinct profiles, both different from the isolates recovered in 1999 - 2000. Two of the 2012 - 2013 farms shared PFGE profiles and had also transported pigs epidemiological connection between these farms was found. Analysis of the number of single nucleotide polymorphisms (SNPs) from the WGS data indicated that the isolates from the farms in 2012 - 2013 were more closely related to each other than to isolates from the outbreak in 1999. It was therefore concluded that the infection was a new introduction and not a persistent infection since the outbreak in 1999. It may further be suggested that there were two or three independent rather than a single introduction. The re-introduction of S. Choleraesuis in Denmark emphasizes the importance of strict hygiene measures in the herds. Further investigations using WGS are now in progress on a larger collection of isolates to study clonality at European level and trace the origin of the infections. 


\section{Introduction}

Pork is one of the most important sources of human foodborne salmonellosis in the EU (EFSA, 2013) and the USA (Gould et al., 2013). Pigs can be colonized with a variety of Salmonella serotypes (EFSA 2008, 2009) but mostly, pigs are asymptomatic carriers. In Denmark, the most common Salmonella serovars in pigs are Salmonella enterica serovar Typhimurium (S. Typhimurium) (including monophasic S. 4,[5],12:i:-), S. Derby and S. Infantis (Argüello et al., 2013, 2014). These serovars may also cause clinical salmonellosis in pigs, but the extent of clinical salmonellosis in pigs in Denmark is uncertain.

S. Choleraesuis is a serovar, which is host-adapted to pigs, and may cause serious outbreaks of salmonellosis and paratyphoid (Griffith et al. 2006). The majority of the $S$. Choleraesuis outbreaks in pigs are caused by var. Kunzendorf (Fedorka-Cray et al., 2000). In the USA, S. Choleraesuis was by far the most frequently found serovar in pigs until the mid-1990'ies. In 1986, 71\% of the isolates from pigs were $S$. Choleraesuis, but thereafter the prevalence of this serovar declined while other serovars increased, and from 1995 and onwards, S. Typhimurium and S. Derby have been most prevalent (Foley et al., 2007). Yet, in 2005 S. Choleraesuis still constituted 9\% of all clinical Salmonella isolates from pigs in the USA (Foley et al., 2007). In Europe, S. Choleraesuis is a relatively rare serovar, both in slaughter pigs and in breeding herds but it has been reported with low frequency in a number of countries (EFSA, 2008, 2009). Out of 42,417 isolates from pigs and pork in 2011, 695 were S. Choleraesuis (EFSA, 2013), but its significance as source of clinical salmonellosis - human or in pigs - is not known.

In the USA, the disease is typically a porcine post weaning disease with septicaemia, enterocolitis and pneumonia and it has been reported to occur most often in farms where pigs of different ages and litters are mixed (Anderson et al., 2000). S. Choleraesuis seems more often to be 
isolated from non-gastrointestinal organs than other serovars, most notably from the lungs (Gray et al., 1996).

In humans, $S$. Choleraesuis tends to be more invasive and cause less gastrointestinal manifestations than most other serotypes and thus, it is a serious infection with a significant mortality (Cohen et al. 1987). Yet, this organism is not a common human pathogen in EU (EFSA, 2013) or in the USA in spite of its relatively high prevalence in American pigs (CDC, 2008). In Denmark, the latest case of human infection with $S$. Choleraesuis was a var. Decatur case in June 2012 and before that a var. Kunzendorf case in December 2011, both travel related (Dr. Eva Møller Nielsen, Statens Serum Institut, Copenhagen, personal communication). However, in Asian countries, such as Thailand and Taiwan, this serovar continues to be important for human illness (Chiu et al., 2004; Hendriksen, 2010), although the incidences seem to be declining (Su et al., 2014).

In Denmark, S. Choleraesuis was last found in pigs at an outbreak in 1999 (Baggesen et al., 2000), but in 2012 and 2013 it reappeared with outbreaks of severe salmonellosis in four farms. It has neither in relation to the outbreak in 1999 (Baggesen et al., 2000) nor to the outbreaks in 2012 and 2013 been possible to identify the primary introduction of infection to the Danish pig herds. This may have been due to limitations in the epidemiological information available but also by an insufficient resolution of isolates by the epidemiological typing methods applied.

In the present study, we describe the reappearance of $S$. Choleraesuis in Danish pig farms during 2012 and 2013. We investigated the clonality of those isolates by the application of pulsed-field gel electrophoresis (PFGE), antimicrobial susceptibility testing (MIC), and whole genome sequencing (WGS), and compared to isolates from the previous Danish outbreak in 1999. 


\section{Material and methods}

\subsection{Farm data Salmonella isolates for epidemiological investigations}

Farm data was retrieved from observations made by the Danish Pig Research Center and registrations via the Salmonella control programme (https://www.retsinformation.dk/Forms/R0710.aspx?id=141725). Data from the serological meat juice surveillance for Salmonella was extracted from the Danish Zoonosis Register. The serological test includes LPS antigens from Salmonella serovars S. Typhimurium and S. Choleraesuis and covers the O factors O1, O4, O5, O6, O7 and O12 (Nielsen et al. 1995). The herds were assigned to one of three infection levels on the basis of serological examination of meat juice samples collected at the slaughterhouse and action was taken for herds reaching levels two or three (Alban et al. 2012). Serological results for the four farms were extracted for the period 2010 - 2014 in order to analyse the time before, during, and after the diagnosis was made in the farms. Other farm data was retrieved from the Central Herd Register (https://chr.fvst.dk). Twenty-three S. Choleraesuis isolates from an outbreak on four pig farms in $2012-2013(n=9)$ and an outbreak in $1999-2000(n=14)$ were included in the study and subjected to PFGE analysis. On the basis of the PFGE results, seven isolates were further analysed using WGS and MIC determination, including three from the outbreak in 1999 - 2000 and one from each of the outbreaks on four different farms in 2012 - 2013.

\section{2. $\quad$ Serotyping and biotyping}

Serotyping was performed by slide agglutination with polyclonal antisera (Statens Serum Institut, Copenhagen, Denmark) according to the White - Kauffmann - Le Minor scheme (Grimont and Weill, 2007) and distinction between $S$. Paratyphi C, S. Typhisuis and the biovars of $S$. 
111

Choleraesuis, var. Kunzendorf and var. Decatur, was performed by biochemical tests (Grimont and Weill, 2007).

\section{3. $\quad$ Pulsed-field gel electrophoresis}

PFGE was carried out according to the PulseNet protocol as previously described (Ribot et al., 2006) using $X b a I$ (Fermentas, Lifesciences) as restriction enzyme and electrophoresis carried out in

a Chef-DR ${ }^{\circledR}$-III $\left(\right.$ Bio-Rad $^{\circledR}$ ). Banding patterns were analysed in BioNumerics ${ }^{\circledR}$ version 7.1 (Applied Maths, Sint-Martens-Latem, Belgium) with a position tolerance of $1.5 \%$ and optimization of 1.5\%. Results were compared using the Dice coefficient for similarity and unweighted pair group method with arithmetic averages (UPMGA) for clustering.

\subsection{Antimicrobial resistance profile}

Antimicrobial susceptibility testing was performed by Minimum Inhibitory Concentration (MIC) determination using a broth microdilution method (SensiTitre system, Trek Diagnostic Systems Ltd., UK) according to recommendations by the Clinical Laboratory Standards Institute (CLSI 2012). Susceptibility was tested against amoxicillin-clavulanic acid, ampicillin, apramycin, cefotaxime, ceftiofur, chloramphenicol, ciprofloxacin, colistin, florfenicol, gentamicin, nalidixic acid, neomycin, spectinomycin, streptomycin, sulphonamides, tetracycline and trimethoprim. MIC values were interpreted using EUCAST epidemiological cut-off values (www.eucast.org) with exception of apramycin for which the value $>16 \mu \mathrm{g} / \mathrm{ml}$ was used (DANMAP, 2012, 2013). 
1.5. Whole genome sequencing, multilocus sequence typing, antimicrobial resistance genes, plasmid replicons, and plasmid multilocus sequence typing

Chromosomal DNA of the subset of six S. Choleraesuis isolates was used to create genomic libraries using the Nextera XT DNA sample preparation kit (Illumina Inc., San Diego, CA, cat. no. FC-131-1024) followed by multiplexed, paired-end sequencing using a MiSeq platform (Illumina). The six selected isolates are marked with an asterisk in Figure 1.

Raw sequence data have been submitted to the European Nucleotide Archive (http://www.ebi.ac.uk/ena) under accession no. PRJEB5487. The fastq files are accessible from the following link: http://www.ebi.ac.uk/ena/data/view/PRJEB5487. The raw reads were assembled using the pipeline available from the Center for Genomic Epidemiology (CGE) (www.genomicepidemiology.org) which is based on Velvet algorithms for de novo short reads assembly (Zerbino and Birney, 2008).

The de novo assembled sequences were analyzed using similar pipelines available on the CGE website. The web-servers, MLST version 1.7, ResFinder version 2.1 and PlasmidFinder version 1.1, available at the Center for Genomic Epidemiology website (www.genomicepidemiology.org) (Zankari et al., 2012; Larsen et al. 2012; Carattoli et al., 2014) were used to identify the multilocus sequence type (ST) for Salmonella enterica, the plasmid replicons, and acquired antimicrobial resistance genes with a selected threshold equal to $85 \%$. The identity and results of the ResFinder were compared with phenotypic antimicrobial susceptibility testing results. Ribosomal MLST (rMLST) types were obtained by querying the rMLST database available at http://pubmlst.org/rmlst/ (Jolley et al., 2012).

\section{6. $\quad$ Single Nucleotide Polymorphisms}


Single nucleotide polymorphisms (SNPs) were determined using the pipeline available on the Center for Genomic Epidemiology (www.genomicepidemiology.org) (Leekitcharoenphon et al., 2012). This pipeline contains various freely available SNP analysis software. Briefly, the paired-end reads from seven $S$. Choleraesuis isolates were aligned against the reference genome, $S$. Choleraesuis strain AE017220. (National Center for Biotechnology Information, accession AE017220, length of 4755700 bp), using Burrows-Wheeler Aligner (BWA) (Li and Durbin, 2009). SAMtools ‘mpileup’ command (Li et al., 2009) and BEDTools (Quinlan and Hall, 2010) were used to determine and filter SNPs. The qualified SNPs were selected once they met the following criteria: (1) a minimum coverage (number of reads mapped to reference positions) of 10; (2) a minimum distance of 15 bps between each SNP; (3) a minimum quality score for each SNP at 30; and (4) all indels were excluded.

The qualified SNPs from each genome were concatenated to a single alignment corresponding to position of the reference genome using an in-house Perl script. In case SNPs were absent in the reference genome, they were interpreted as not being a variation and the relative base from the reference genome was expected (Leekitcharoenphon et al., 2012). The concatenated sequences were subjected to multiple alignments using MUSCLE from MEGA5 (Tamura et al., 2011). The final phylogenetic SNP tree was computed by MEGA5 using the maximum likelihood method (Tamura and Nei, 1993) of 1,000 bootstrap replicates (Felsenstein, 1985).

\section{Results}

\subsection{Outbreak description}

The outbreaks in 2012 and 2013 occurred on four different farms, arbitrarily designated farm A, B, C, and D. In addition, isolates for the investigations from an outbreak in 1999 on a farm, 
designated farm E, were included in the typing studies. The location of the five farms is shown in Figure 1 together with some information about each farm.

Farm A experienced a dramatic increase in Salmonella sero-prevalence in meat juice samples in August 2012 and became classified as a level 2 herd in September and level 3 in October (Fig 2) Concomitantly, there was an outbreak of oedema disease, and the mortality increased to appr. $20 \%$ among pigs up to $30 \mathrm{~kg}$. Antibiotic treatment was initiated at this point, but unfortunately, no samples were taken for Salmonella culture. The farm had previously in January 2011 been diagnosed with $S$. Infantis. It was not until November 2012 that carcasses were submitted to a laboratory and $S$. Choleraesuis was identified from lung tissue. The pigs were subsequently culled and the premises cleaned and disinfected, and in February 2013 new SPF animals were installed. Pen floor samples collected in January 2014 to follow up on Salmonella status did not show presence of $S$. Choleraesuis, but $S$. Infantis was still present. Serological data showed that the herd continued to have a low proportion of sero-positive samples (Figure 2) - possibly due to $S$. Infantis.

Farm B purchased pigs from farm A during the summer 2012, and when farm A was diagnosed with S. Choleraesuis, farm B was also investigated due to the registered transfer of pig. At that time, the Salmonela seroprevalence in the herd had changed from zero to low and the farm had experienced increased mortality, and upon laboratory investigation of dead pigs, the farm was diagnosed with S. Choleraesuis as well as S. Derby in January 2013. The herd was then culled; some of the oldest buildings were destroyed, and the remaining were cleaned and disinfected, where after the farm started to operate again. Follow-up pen floor samples collected in September 2013 did not show presence of $S$. Choleraesuis but both $S$. Derby and $S$. Infantis were found. Serological data showed that a moderate proportion of sero-positive samples was still present (Figure 2). 
Farm C was a family operated farm with no foreign assistance. There was a general very high mortality around $20-30 \%$ among the $7-50 \mathrm{~kg}$ pigs. For at least a couple of years prior to the diagnosis, the herd had a high sero-prevalence for Salmonella in meat juice samples. Forty-one pigs were in the spring 2013 sold to another herd, which subsequently experienced very high meat juice sero-prevalence and became categorised as a level 3 herd. Therefore a trace back from that farm to the supplier herd, farm C, was made and $S$. Choleraesuis was cultured in pen floor samples there in August 2013. The farm had no known previous culture confirmed history of Salmonella. The farm, which had purchased the 41 pigs from farm C, was not tested for Salmonella by culture, because the owner decided to empty the farm soon after the diagnosis was made in farm C. The buildings were then cleaned and disinfected before new pigs were installed. That farm has subsequently been tested but $S$. Choleraesuis has not been found. Farm C is operating as before and with $S$. Choleraesuis probably still present. Some management procedures have been changed to reduce infection and contamination within herd and there have been repeated antibiotic treatments to reduce mortality. Serological data of meat juice samples showed a very high sero-prevalence for some months followed by a dramatic decrease, and after July 2014 - at least until November - no seropositive samples have been detected.

Farm D purchases pigs for on-growing from a single sow herd, which supplies pigs to several other herds. Neither the supplying sow herd nor any of the other herds receiving pigs from that supplier herd are or have been tested positive with $S$. Choleraesuis. Farm D had experienced high Salmonella sero-prevalence since October 2012 (Figure 2) and there was a general, very high mortality, approximately 20 - $30 \%$, among 30 - 50 kg pigs. There was no known history of Salmonella. In December 2013 dead pigs were submitted to a laboratory with an anamnesis of diarrhoea, respiratory problems and septicaemia, and $S$. Choleraesuis was cultured from the pigs. Clinical salmonellosis is subjected to antibiotic treatment, but mortality is still high and it is still 
infected with $S$. Choleraesuis. Moderate to high proportions of sero-positive meat juice samples are still detected in the herd (Figure 2)

Both farm C and D have purchased corn directly from Eastern Europe, delivered on site by truck, without involving a feed company and without heat treatment.

The outbreak on farm E in 1999 has been described elsewhere (Baggesen et al., 2000) and will not be further dealt with here.

\section{2. $\quad$ Pulsed-field gel electrophoresis}

A total of five different PFGE patterns were recognized among the 23 isolates (Figure 3). The 14 isolates from the outbreak on farm E in 1999 - 2000 displayed three different profiles (containing 4, 9, and 1 isolate, respectively), but they clustered together as they deviated in only one or two bands. The five isolates from the two farms A and B that were recovered from the outbreaks in 2012 - 2013, had identical PFGE profiles. These two farms were also epidemiologically connected, i.e. farm A had delivered pigs to farm B, as described above. The three isolates from farm C and the isolate from farm D recovered from outbreaks in 2013 were indistinguishable (Figure 3).

\subsection{Whole genome sequencing}

On the basis of the WGS data, all tested isolates were found to have MLST ST1804, a type which has not previously been reported in the PubMLST database

(http://mlst.ucc.ie/mlst/dbs/Senterica). rMLST divided the isolates into two groups. The isolates 
from 2012 - 2013 belonged to ST3723 and the isolates from 1999 to ST3636, differing by a single nucleotide mismatch in one of the alleles (Figure 4).

A total of 672 SNPs were identified and used to construct a phylogenetic tree (Figure 4). None of the Danish isolates from 1999 - 2000 and 2012 - 2013 were identical but clustered together in two groups. The isolates from 1999 - 2000 had less than 93 SNPs difference between them, while the isolates from 2012 - 2013 separated in two subgroups with 23 and 134 SNPs difference between the isolates, respectively. The two isolates from farm A and B, which were epidemiologically related, had only 23 SNPs difference, indicating a close relatedness. This was underlined by the fact that they also had identical PFGE profiles. The two other isolates, farm $\mathrm{C}$ and D, had more SNPs difference, indicating that they were less closely related, although they shared the same PFGE profile but different from that of farm A and B.

To further characterize the isolates WGS data were analysed for the content of plasmid replicons, and acquired antimicrobial resistance genes. All sequenced isolates contained plasmid replicons of the incFIB and incFII type. The three isolates from 2012 - 2013 in addition, carried plasmids of replicon type incQ1, while the isolates from 1999 had three different replicon types (Figure 4).

\subsection{Antimicrobial susceptibility}

The phenotypic antimicrobial susceptibility of the seven isolates obtained by MIC determination is shown in Figure 3. The four isolates from the outbreaks in 2012 - 2013 shared the same resistance profile, being resistant to sulphonamides and streptomycin. The three isolates from 1999 had different resistance profiles: one was resistant only to streptomycin whereas the two others were 
multi-resistant to streptomycin, spectinomycin, sulphonamides and tetracyclines, and one of them additionally to trimethoprim (Figure 4).

The MIC results corresponded well for most of the isolates with the contents of antimicrobial resistance genes, which were identified from the WGS data. No resistance genes were shared between isolates from 1999 - 2000 and isolates from 2012 - 2013 (Figure 4).

\section{Discussion}

Most often pigs are symptomless carriers of Salmonella, although S. Typhimurium (including monophasic variants) and occasionally other serovars including $S$. Infantis and $S$. Derby may cause clinical salmonellosis with diarrhoea as primary clinical symptom. In contrast, diarrhoea is less pronounced among pigs infected with $S$. Choleraesuis. Clinical symptoms here usually include fever, inappetence, lethargy, and respiratory symptoms with coughing and difficulties breathing. Symptoms often appear 24 - 36 hours post infection, and gastrointestinal symptoms usually appear after 4 - 5 days (Fedorka-Cray et al. 2000). S. Choleraesuis may be dormant in a pig farm but then be activated by stress factors, such as porcine circovirus (PCV2) or porcine reproductive and respiratory syndrome (PRRS) virus outbreaks (Chiu et al., 2004). In the Danish herds in 2012 2013 there was a concurrent outbreak of oedema disease in farm A, and both farm C and D had several concurrent disease problems with high mortality. It can only be suggested whether this has contributed to the severity of the $S$. Choleraesuis outbreaks in these herds or vice versa, but the occurrence of other diseases at the same time has likely delayed the diagnosis of $S$. Choleraesuis, because the herds were treated for the infections that were known to be present, and for a long time no further laboratory investigation was made to look for other pathogens. Huang et al. (2009) found high levels of resistance to ampicillin, tetracycline and ticarcillin, but low resistance to 
spectinomycin and no resistance to other tested compounds in a collection of American isolates. In general, from European countries and the USA, there are not many reports on antimicrobial susceptibility of this serovar. In the present study, we fortunately found only low levels of resistance, i.e. the isolates from 2012 - 2013 were only resistant to streptomycin and sulfonamides, while the isolates from the 1999 outbreak were resistant to more compounds. Notably, critical resistance to fluoroquinolones or cephalosporins was not found.

The source of the infection in the Danish herds is presently unknown. Apart from the trade of pigs from farm $\mathrm{A}$ to $\mathrm{B}$, there are no common factors that connect them, and they are located geographically distant from each other (Figure 1). It is generally accepted that $S$. Choleraesuis is rarely found in feed or in animals other than pigs, and that the source is consequently most often limited to horizontal transfer by carrier pigs (Anderson et al. 2000). Experimental infection studies in two days old pigs have shown that some of the pigs were shedding the bacterium for at least 85 days (Anderson et al. 2000), during which period they are potentially able to transfer the infection, which is readily transmitted to uninfected pigs via contact with infected animals or their faeces (Gray et al. 1996). In the present cases from 2012 - 2013, no pigs were imported into three of the herds, which rules out carrier pigs as source in these herds. One herd (farm B) had received pigs from one of the other herds (farm A), and since the PFGE patterns of isolates from these two connected herds were identical and the SNP tree also showed close relatedness, it is concluded that the infection was transferred from one herd to the other. Farm A is a new and well managed SPF herd with a strict biosecurity and no entry of animals. It has not been possible to identify any potential sources or routes of infection. The isolates from Farm C and D had indistinguishable PFGE patterns and both farms had very poor biosecurity. Farm C had not received any pigs from other farms, and farm D had only purchased pigs from a single supplier, which was not detected with $S$. Choleraesuis infection. Therefore, it is concluded that live pigs were not the source of 
infection in any of the herds, except for farm B. S. Choleraesuis has been found in wild boars in Europe, at least in Italy (Chiari et al., 2013; Zottola et al., 2013) and Spain (Perez et al., 1999), which suggests a wildlife reservoir that may spill over to farmed pigs or vice versa. In Denmark there is not a stock of wild boars so, although wild boars from Germany occasionally cross the border, this source of infection can probably be ruled out.

Although the analyses here do not address where the infections came from, the typing data together with the epidemiological information from the farms suggest that the outbreaks in 2012 2013 may have been caused by two or maybe three separate introductions rather than a single event. This theory is supported by the fact that two different PFGE profiles were involved, and that the SNP analysis grouped the isolates in two groups. On the other hand, all isolates had identical MST types, resistance profiles, plasmid replicons and rMLST profiles, indicating that they were closely related. At the outbreak in 1999 - 2000, three different PFGE profiles were found on the same farm, which indicates some variability within this serovar. It therefore seems likely that there was some kind of connection between the outbreaks although epidemiological investigations have not been able to point out any common factors. It can only be speculated how the infection was brought to the country. There is a considerable export of live pigs from Denmark to especially Germany but also other European countries, and it is possible that the trucks returning to Denmark may occasionally not have been properly cleaned and disinfected. Two of the farms had imported corn directly delivered by truck from an area of Europe where $S$. Choleraesuis is endemic. Feed is known occasionally to be contaminated with Salmonella, and may be a risk factor for introduction of a plethora of Salmonella serovars into animal herds (Hald et al. 2012), but to the authors’ knowledge, $S$. Choleraesuis has never been found in animal feed in Denmark. Yet, it cannot be entirely ruled out that such feed shipments may have been contaminated with the bacterium. However, the four farms all had different suppliers of feed, so it can be excluded that there was a 
common source of entry from feed. Salmonella survives well in the environment. Although $S$.

Choleraesuis is host adapted and often believed not to survive well outside a host, experiments have shown that it is able to survive in faeces from infected pigs for at least 13 months and be infective for at least 4 months (Gray and Fedorka-Cray 2001). Therefore, hygiene and biosecurity measures are extremely important for prevention of Salmonella in pigs and care must be taken to clean and disinfect equipment, etc., and not allow faecal contaminated equipment to enter. Care must in particular be taken when pigs are collected by trucks for sale or slaughter, or when trucks deliver feed to the farm that no faecal material or contaminated equipment from the truck is introduced.

Although humans have not been described to be carriers of $S$. Choleraesuis, humans have been implicated as passive vectors, contributing to the spread between animals and herds (Wolf et al, 2011). In the present cases there is no evidence that humans have been the source or vector of the infection, as three of the farms had no foreign employees.

In conclusion: S. Choleraesuis was reintroduced in four Danish pig herds in 2012 - 2013 after 12 years absence, and increasing meat juice sero-prevalence against Salmonella was detected in the herds prior to isolation of $S$. Choleraesuis. In spite of intensive molecular typing, the sources of infection could not be traced on the basis of the current investigation. The infections were likely not brought by live animals or humans but direct imported feed cannot be excluded as source. The results suggest that it was two or three independent introductions but typing data indicated that the isolates were related and ongoing WGS on a larger collection of $S$. Choleraesuis from many countries may allow better conclusions on potential sources to be drawn.

\section{Acknowledgement}



361 for providing the sequencing data. 


\section{References}

Alban, L., Baptista, F.M., Møgelmose, V., Sørensen, L.L., Christensen, H., Aabo, S., Dahl, J., 2012. Salmonella surveillance and control for finisher pigs and pork in Denmark — A case study. Food Research International 45, 656-665.

Anderson, R.C., Genovese, K.J., Harvey, R.B., Stanker, L.H., DeLoach, J.R., Nisbet, D.J., 2000. Assessment of the long-term shedding pattern of Salmonella serovar choleraesuis following experimental infection of neonatal piglets. J. Vet. Diagn. Invest. 12, 257-260.

Argüello, H., Sørensen, G., Carvajal, A., Baggesen, D.L., Rubio, P., Pedersen, K, 2013. Prevalence, serotypes and resistance patterns of Salmonella in Danish pig production. Res. Vet. Sci. 95, 334-342.

Argüello, H., Sørensen, G., Carvajal, A., Baggesen, D.L., Rubio, P., Pedersen, K., 2014. Characterization of the emerging Salmonella 4,[5],12:i:- in Danish animal production. Foodborne Pathog. Dis. 11, 366-372.

Baggesen, D.L., Christensen, J., Jensen, T.K., Skov, M.N., Sørensen, G., Sørensen, V., 2000. Udbrud af Salmonella enterica subsp. enterica serovar Choleraesuis var. Kunzendorf (S. Choleraesuia) i en dansk svinebesætning. Dansk Veterinærtidsskrift 83, 6-12.

CDC, 2008. Salmonella surveillance: annual summary, 2006. CDC, Department of Health and Human Services, Atlanta, GA.

Chiari, M., Zanoni, M., Tagliabue, S., Lavazza, A., Alborali, L.G., 2013. Salmonella serotypes in wild boars (Sus scrofa) hunted in northern Italy. Acta Vet. Scand. 55, 42. http://www.actavetscand.com/content/55/1/42 
Chiu, C.-H., Su, L.-H., Chu, C., 2004. Salmonella enterica serotype Choleraesuis: Epidemiology, pathogenesis, clinical disease, and treatment. Clin. Microbiol. Rev. 17, 311-322. doi: 10.1128/CMR.17.2.311-322.2004.

Clinical Laboratory Standards Institute, 2012. Methods for dilution antimicrobial susceptibility tests for bacteria that grow aerobically; Approved Standard—Ninth Edition. CLSI document M7A9. CLSI, Wayne, PA, USA.

Cohen, J.L., Bartlett, J.A., Corey, R., 1987. Extra-intestinal manifestations of Salmonella infections. Medicine (Baltimore) 66, 349-388.

DANMAP 2012, 2013. Use of Antimicrobial Agents and Occurrence of Antimicrobial Resistance in Bacteria from Food Animals, Foods and Humans in Denmark. http://www.DANMAP.org

EFSA, 2008. Report of the Task Force on Zoonoses Data Collection on the analysis of the baseline survey on the prevalence of Salmonella in slaughter pigs, in the EU, 2006-20071. Part A: Salmonella prevalence estimates. The EFSA Journal 135, 1-111.

EFSA, 2009. Analysis of the baseline survey on the prevalence of Salmonella in holdings with breeding pigs, in the EU, 2008, Part A: Salmonella prevalence estimates, EFSA Journal 7(12): [93 pp.]. doi:10.2903.1377.

EFSA, 2013. The European Union Summary Report on Trends and Sources of Zoonoses, Zoonotic Agents and Food-borne Outbreaks in 2011. EFSA Journal 11: 3129, 1-250.

Fedorka-Cray, P.J., Gray, J.T., Wray, C, 2000. Salmonella infections in pigs. In: C. Wray, A. Wray (eds.): Salmonella in domestic animals. CAB International, Oxon, UK, pp. 191-207. 
404

405

406

407

408

409

410

411

412

413

414

415

Felsenstein, J., 1985. Confidence limits on phylogenies: An approach using the bootstrap. Evolution 39; 783-791.

Foley, S.L., Lynne, A.M., Nayak, R., 2008. Salmonella challenges: prevalence in swine and poultry and potential pathogenicity of such isolates. J. Anim. Sci. 86(14 Suppl), E149-62.

Gould, L.H., Walsh, K.A., Vieira, A.R., Herman, K., Williams, I.T., Hall, A.J., Cole, D., 2013. Surveillance for Foodborne Disease Outbreaks — United States, 1998-2008. Morbidity and Mortality Weekly Report 62(SS02), 1-34.

http://www.cdc.gov/mmwr/preview/mmwrhtml/ss6202a1.htm

Gray, J.T., Fedorka-Cray, P.J., 2001. Long-term survival and infectivity of Salmonella choleraesuis. Berl. Munch. Tierarztl. Wochenschr. 114, 370-374.

Gray, J.T., Fedorka-Cray, P.J., Stabel, T.J., Kramer, T.T., 1996. Natural transmission of Salmonella choleraesuis in swine. Appl. Environ. Microbiol. 62, 141-146.

Griffith, R.W., Schwartz, K.J., Meyerholz, D.K., 2006. Salmonella. In: Straw, B.E., Zimmerman, J.J., D’Allaire, S., Taylor, D.J. (eds.): Diseases in swine. $9^{\text {th }}$ ed. Blackwell Publishing, Oxford, UK, pp. 739-754

Grimont, P.A.D., Weill, F.X., 2007. Antigenic formulae of the Salmonella serovars. $9^{\text {th }}$ ed., Institut Pasteur, Paris.

Hald, T., Wingstrand, A., Pires, S.M., Vieira, A., Domingues, A.R., Lundsby, K., Dalhoff Andersen, V., Thrane, C., 2012. Assessment of the human-health impact of Salmonella in animal feed. National Food Institute, Technical University of Denmark, http://www.google.dk/url?sa=t\&rct=j\&q=\&esrc=s\&frm=1\&source=web\&cd=2\&ved=0CC4QF jAB\&url=http\%3A\%2F\%2Fwww.food.dtu.dk\%2F \%2Fmedia\%2FInstitutter\%2FFoedevareins 
tituttet\%2FPublikationer\%2FPub-2013\%2FReport-Assessment-of-the-human-health-impactof-Salmonella-in-animalfeed.ashx\&ei=MkoYU9bvGYeP5ASu7IEQ\&usg=AFQjCNGHV2NBYA3ZXrjot3uNTzSprdrtJ $\underline{\text { Q\&bvm=bv.62577051,d.bGE }}$

Hendriksen, R., 2010. Global epidemiology of non-typhoidal Salmonella infections in humans. Ph.D. thesis, Technical University of Denmark, Kongens Lyngby, Denmark.

Huang, T.M., Lin, T.L., Wu, C.C., 2009. Serovar distribution and antimicrobial susceptibility of swine Salmonella isolates from clinically ill pigs in diagnostic submissions from Indiana in the United States. Lett. Appl. Microbiol. 48, 331-336. doi: 10.1111/j.1472-765X.2008.02530.x.

Jolley, K. A., Bliss, C. M., Bennett, J. S., Bratcher, H. B., Brehony, C., Colles, F. M., Wimalarathna, H., Harrison, O. B., Sheppard, S. K., Cody, A.J., Maiden, M.C.J., 2012. Ribosomal multilocus sequence typing: universal characterization of bacteria from domain to strain. Microbiology 158, 1005-1015.

Leekitcharoenphon, P., Kaas, R.S., Thomsen, M.C., Friis, C., Rasmussen, S., Aarestrup, F.M., 2012. snpTree - a web-server to identify and construct SNP trees from whole genome sequence data. BMC Genomics 13, Suppl 7:S6. http://www.biomedcentral.com/1471-2164/13/S7/S6

Li, H., Durbin, R., 2009. Fast and accurate short read alignment with Burrows-Wheeler transform. Bioinformatics 25, 1754-1760.

Li, H., Handsaker, B., Wysoker, A., Fennell, T., Ruan, J., Homer, N., Marth, G., Abecasis, G., Durbin, R., 1000 Genome Project Data Processing Subgroup, 2009. The Sequence Alignment/Map format and SAMtools. Bioinformatics 25, 2078-2079. doi: 10.1093/bioinformatics/btp352. 
Nielsen, B.; Baggesen, D. L., Bager, F., Haugegaard, J., Lind, P., 1995. The serological response to Salmonella serovars typhimurium and infantis in experimentally infected pigs. The time course followed with an indirect anti-LPS ELISA and bacteriological examinations. Veterinary Microbiology 47, 205-218.

Perez, J., Astorga, R., Carrasco, L., Mendes, A., Perea, A., Sierra, M.A., 1999. Outbreak of salmonellosis in farmed European wild boars (Sus scrofa ferus). Vet. Rec. 145, 464-465.

Quinlan, A.R., Hall, I.M., 2010. BEDTools: a flexible suite of utilities for comparing genomic features. Bioinformatics 26, 841-842.

Ribot, E.M., Fair, M.A., Gautom, R., Cameron, D.N., Hunter, S.B., Swaminathan, B., Barrett, T.J., 2006. Standardization of pulsed-field gel electrophoresis protocols for the subtyping of Escherichia coli O157:H7, Salmonella, and Shigella for PulseNet. Foodborne Pathog Dis. 3, 59-67.

Su, L.H., Wu, T.L., Chiu, C.H., 2014. Decline of Salmonella enterica serotype Choleraesuis infections, Taiwan [letter]. Emerg. Infect. Dis. 20, 715-716. http://dx.doi.org/10.3201/eid2004.130240

Tamura, K., Nei, M., 1993. Estimation of the number of nucleotide substitutions in the control region of mitochondrial DNA in humans and chimpanzees. Mol. Biol. Evol. 10, 512-526.

Tamura, K., Peterson, D., Peterson, N., Stecher, G., Nei, M., Kumar, S., 2011. MEGA5: molecular evolutionary genetics analysis using maximum likelihood, evolutionary distance, and maximum parsimony methods. Mol. Biol. Evol. 28,2731-2739. doi: 10.1093/molbev/msr121. 
Wegener, H.C., Hald, T., Lo Fo Wong, D., Madsen, M., Korsgaard, H., Bager, F., Gerner-Smidt, P., Mølbak, K., 2003. Salmonella control programs in Denmark. Emerg. Infect. Dis. 2003; 9: 774780.

Wolf, T.M., Wünschmann, A., Morningstar-Shaw, B., Pantlin, G.C., Rasmussen, J.M., Thompson, R.L., 2011. An outbreak of Salmonella enterica serotype Choleraesuis in goitered gazelle (Gazella subgutrosa subgutrosa) and a Malayan tapir (Tapirus indicus). J. Zoo. Wildl. Med. 42, 694-699.

Zerbino, D.R., Birney, E., 2008. Velvet: algorithms for de novo short read assembly using de Bruijn graphs. Genome Res. 18, 821-829.

Zottola, T., Montagnaro, S., Magnapera, C., Sasso, S., De Martino, L., Bragagnolo, A., D'Amici, L., Condoleo, R., Pisanelli, G., Iovane, G., Pagnini, U., 2013. Prevalence and antimicrobial susceptibility of Salmonella in European wild boar (Sus scrofa); Latium Region - Italy. Comp. Immunol. Microbiol. Infect. Dis. 36, 161-168. doi: 10.1016/j.cimid.2012.11.004. 
Figure 1. Approximate location of the Danish farms infected with $S$. Choleraesuis and general farm information. Data for farm E have been described elsewhere (Baggesen et al. 2000).

Figure 2. Data for Salmonella sero-prevalences on the four outbreak farms together with detection are included.

Figure 3. Dendrogram of 23 Danish isolates of $S$. Choleraesuis from five pig farm outbreaks produced from pulsed-field gel electrophoresis results. Isolates marked with an asterisk were subjected to whole genome sequencing.

Figure 4. SNP tree together with results for the antimicrobial susceptibility tests, presence of 499 antimicrobial resistance genes and plasmids for the seven sequenced isolates of $S$. Choleraesuis 500 from Danish pig farms. SNP differences between branches are indicated with numbers in the 501 dendrogram. The total no. of SNPs was 672. Str = streptomycin, Sul = sulfonamides, Spe $=$ spectinomycin, Tet $=$ tetracyclines, $\mathrm{Tmp}=$ trimethoprim. $\mathrm{ND}=$ none detected, $\mathrm{ST}=$ sequence type. 


\section{Farm A}

Diagnosis: December 2012

Facility: modern, built 2008

Herd size: 5250 pigs, including 650

sows

Biosecurity: high. Specific pathogen free (SPF) herd

Production: produces slaughter pigs and sells pigs for on-growing and export. No pigs are bought to the

fっum

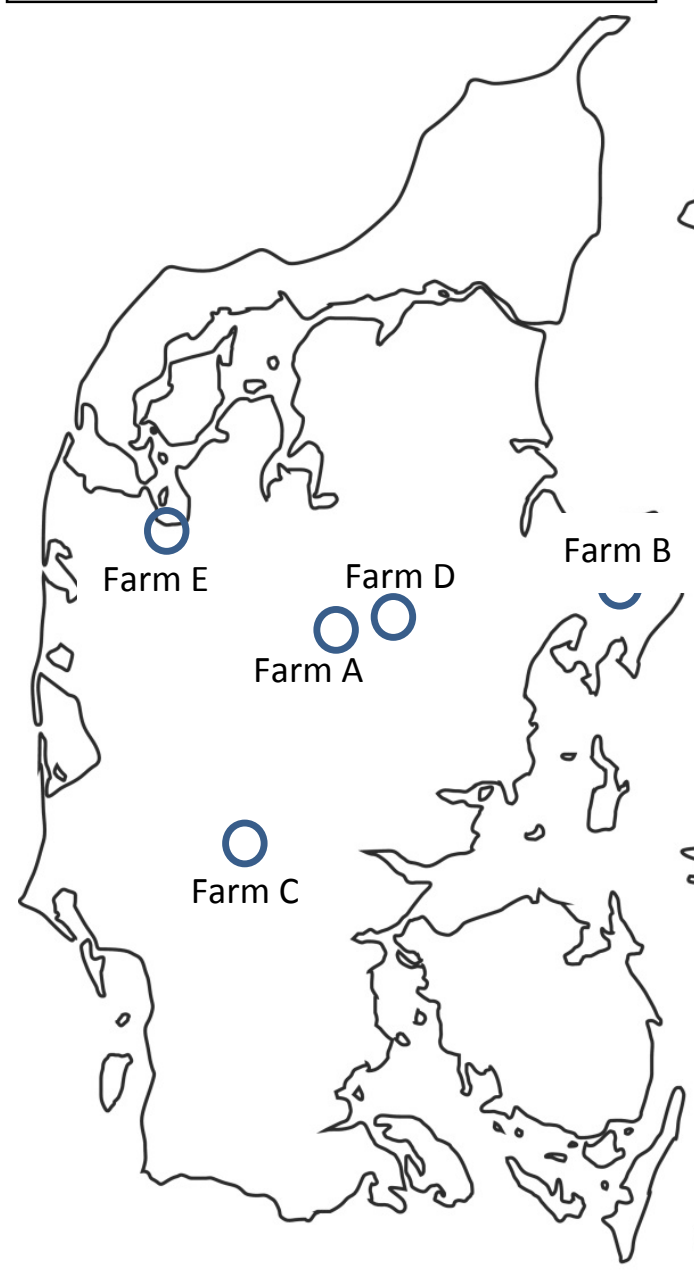

\section{Farm D}

Diagnosis: December 2013

Facility: old buildings

Herd size: 3000 pigs

Biosecurity: poor

Production: only slaughter pig

purchased from a single sow herd

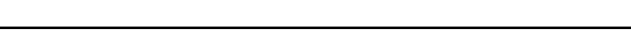

$\nabla \quad$\begin{tabular}{|l|}
\hline Farm B \\
Diagnosis: January 2013 \\
Facility: old buildings \\
Herd size: 2400 pigs \\
Biosecurity: poor \\
Production: only slaughter pigs. \\
Purchased from several suppliers, \\
inclı idino farm $\Delta$
\end{tabular}

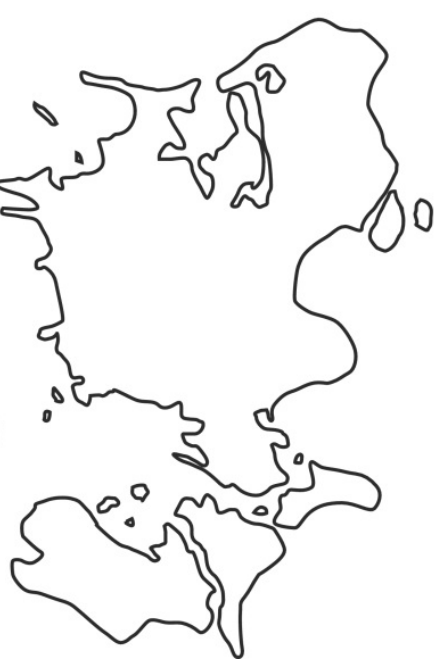

\section{Farm C}

Diagnosis: August 2013

Facility: old buildings

Herd size: 2410 pigs, including 220

sows

Biosecurity: very poor

Production: produces slaughter pigs

and collc nioc for nnornwino No nioc 


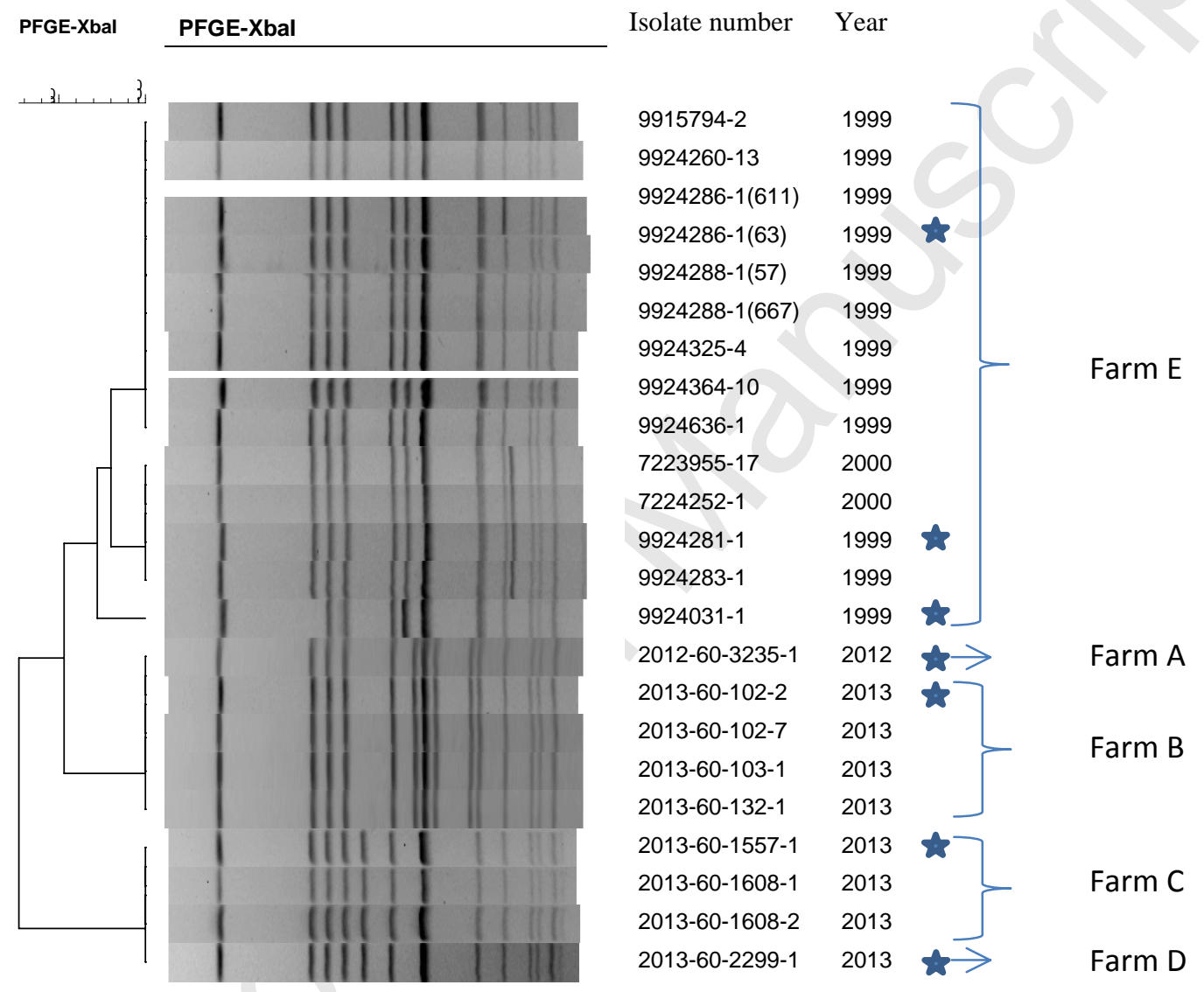




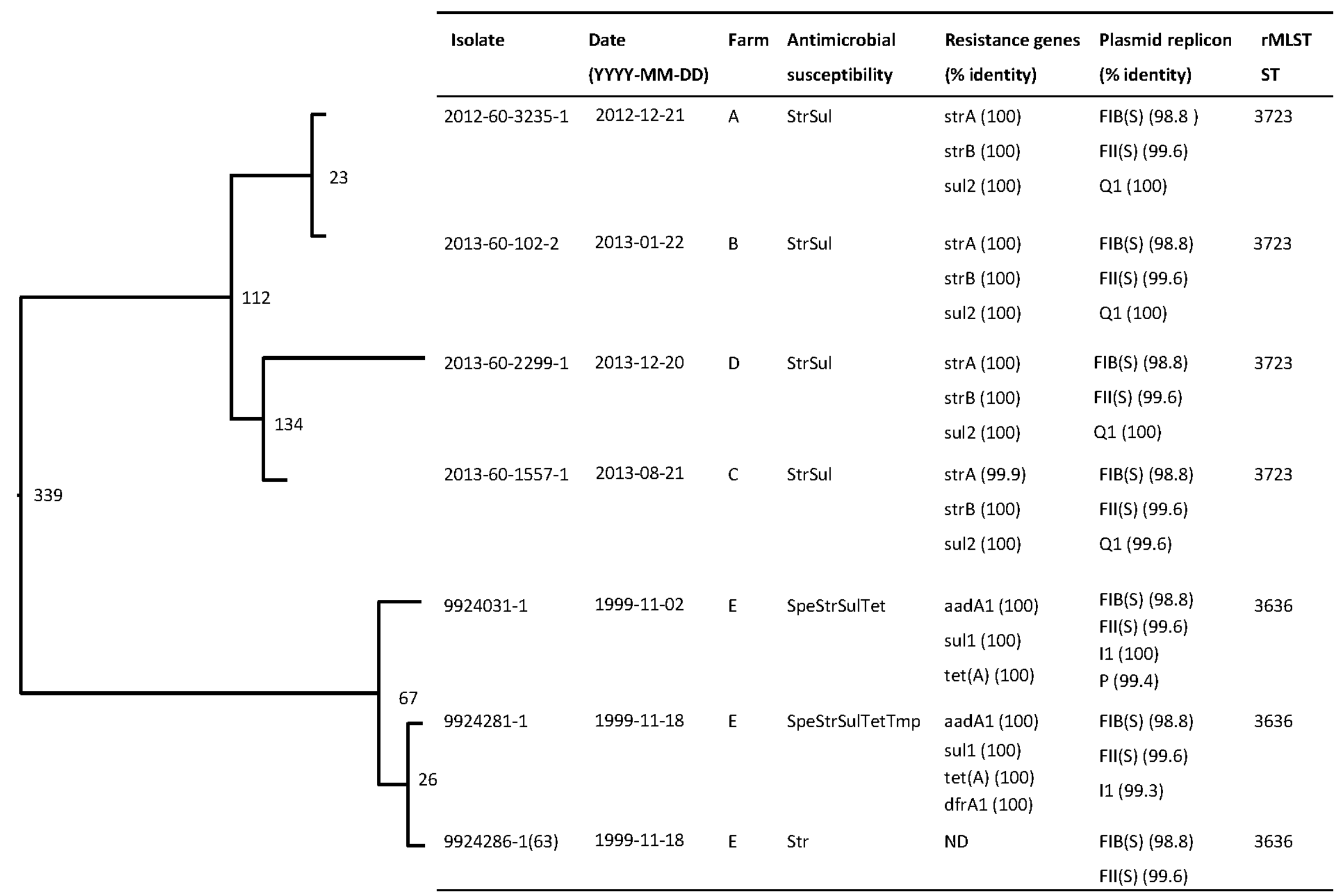


Highlights

- Salmonella Choleraesuis reappeared in Danish pig herds in 2012 - 2013

- Outbreaks were preceded by increased meat juice sero-prevalence

- Severe disease problems occurred in affected herds

- Two or three independent introductions occurred based on molecular typing and epidemiology

- Sources of the infection could not be established 

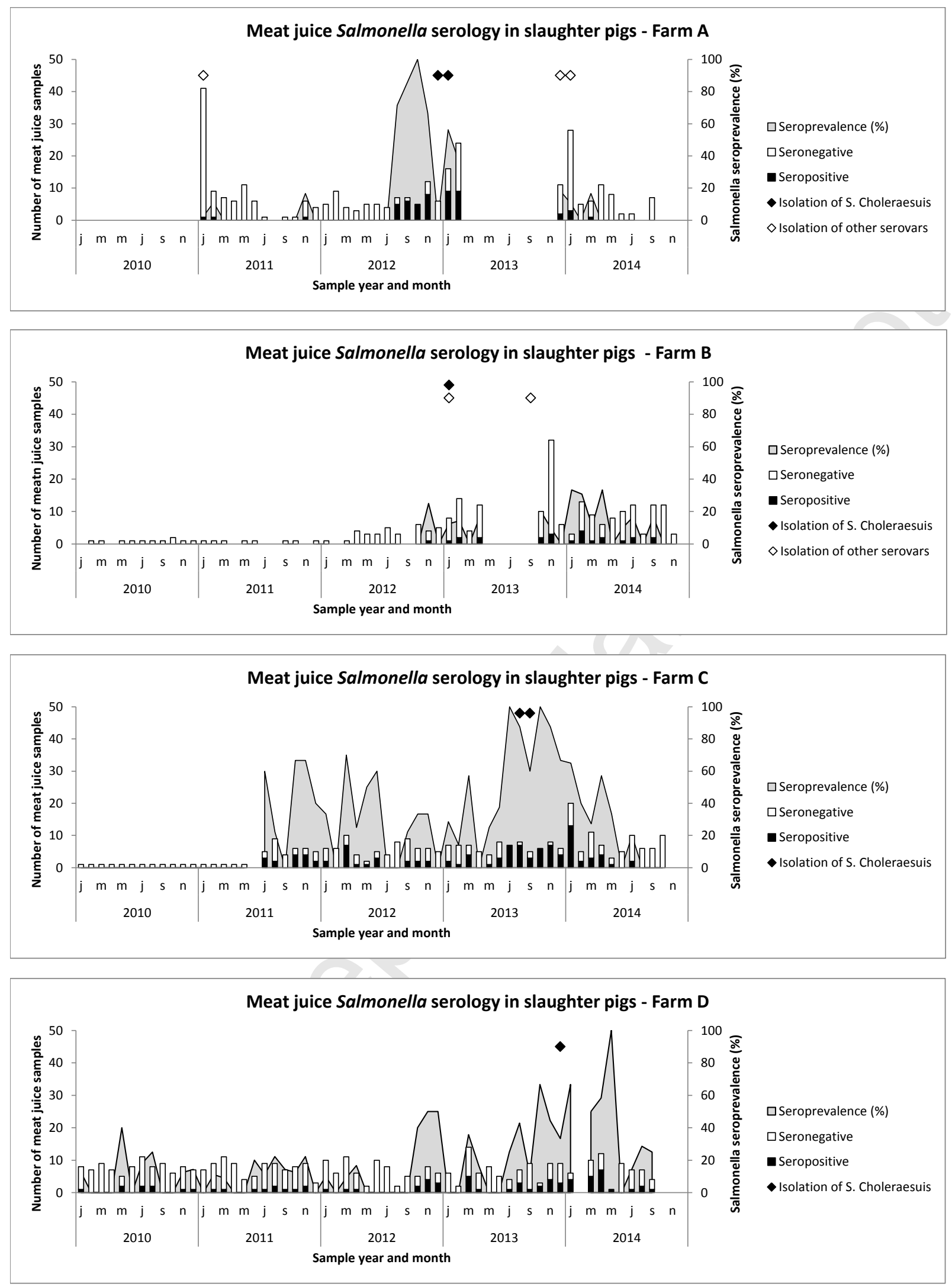